\title{
Diversity and distribution of Blastocystis sp. subtypes in non-human primates
}

\author{
MOHAMMED A. ALFELLANI ${ }^{1,9}$, ALISON S. JACOB ${ }^{1}$, NATALI ORTIZ PEREA ${ }^{1}$, \\ ROSINA C. KRECEK ${ }^{2,3}$, DERYA TANER-MULLA ${ }^{1}$, JACO J. VERWEIJ ${ }^{4 \dagger}$, \\ BRUNO LEVECKE ${ }^{5,6}$, EGBERT TANNICH$^{7}$, C. GRAHAM CLARK $^{1}$ \\ and $\mathrm{C}$. RUNE STENSVOLD ${ }^{8 *}$ \\ ${ }^{1}$ Faculty of Infectious and Tropical Diseases, London School of Hygiene and Tropical Medicine, London, UK \\ ${ }^{2}$ Department of Research, Ross University School of Veterinary Medicine (RUSVM), Basseterre, St. Kitts \\ ${ }^{3}$ Department of Zoology, University of Yohannesburg, Auckland Park, South Africa \\ ${ }^{4}$ Department of Parasitology, Leiden University Medical Centre, the Netherlands \\ ${ }^{5}$ Laboratory for Parasitology, Faculty of Veterinary Medicine, Ghent University, Ghent, Belgium \\ ${ }^{6}$ Centre for Research and Conservation, Royal Zoological Society of Antwerp, Antwerp, Belgium \\ ${ }^{7}$ Bernhard-Nocht-Institut für Tropenmedizin, Hamburg, Germany \\ ${ }^{8}$ Department of Microbiology and Infection Control, Statens Serum Institut, Copenhagen, Denmark \\ ${ }^{9}$ Department of Parasitology, Faculty of Medicine, Sebha University, Sebha, Libya
}

(Received 10 October 2012; revised 6 December 2012 and 15 fanuary 2013; accepted 22 February 2013)

S UMMAR Y

Blastocystis SSU-rDNA sequence data from 317 captive and free-living non-human primates (NHPs) representing 30 genera of apes, Old and New World (OW and NW) monkeys and prosimians were analysed to investigate subtype (ST) and allele distribution among hosts. Excluding 20 mixed ST infections, 27\% of the sequences belonged to ST1, 22\% to ST2, $34 \%$ to ST3, $1 \%$ to ST $4,4 \%$ to ST5, $11 \%$ to ST $8,<1 \%$ to ST 13 and $1 \%$ to ST 15 . The study confirmed cryptic host specificity of ST1 and ST3; conversely, considerable overlap in ST2 alleles exists among humans and NHPs. Subtype distribution in humans and NHPs differs mainly in that ST4 is rarely reported in NHPs while ST5 and ST8 are both unusual in humans. This may be due to host specificity and/or the apparent geographically restricted range of some subtypes. While the distribution of ST1, ST2 and ST3 was independent of NHP group or geographical association, ST5 was seen only in apes and OW monkeys and ST8 primarily in arboreal NHPs and only in species native to Asia or South America.

Key words: Blastocystis, non-human primates, host range, genetic diversity.

\section{INTRODUCTION}

The most common non-fungal eukaryotic organism in the intestine of humans and possibly non-human primates (NHPs) is Blastocystis. This genus comprises many significantly divergent small-subunit ribosomal RNA gene (SSU-rDNA) lineages, termed subtypes (ST), and in humans, NHPs, other mammals and birds, 14 have already been defined (Stensvold et al. 2007, 2009; Parkar et al. 2010; Fayer et al. 2012). In other taxa, Blastocystis STs would probably be considered distinct species. There is substantial overlap in the range of Blastocystis STs reported from humans and from other mammals and birds, which has prompted some authors to propose that zoonotic transmission is the origin of many human infections (e.g. Parkar et al. 2007, 2010; Stensvold et al. 2008).

* Corresponding author: Department of Microbiology and Infection Control, Statens Serum Institut, Copenhagen, Artillerivej 5, DK-2300 Copenhagen S, Denmark. E-mail: run@ssi.dk

$\uparrow$ Current address: Laboratory for Medical Microbiology and Immunology, Laboratory for Clinical Pathology, St. Elisabeth Hospital, Tilburg, the Netherlands.
While ST and prevalence data for Blastocystis in humans are accumulating quickly from numerous studies, we still know relatively little about the distribution, host specificity and genetic variation in STs found in other hosts, including NHPs. Perhaps not surprisingly, studies have indicated that there is great similarity in the STs infecting humans and NHPs, with ST1, ST2 and ST3 predominating (Parkar et al. 2007, 2010; Yoshikawa et al. 2009; Petrášová et al. 2011). By studying Blastocystis from humans and NHPs on Rubondo Island, Tanzania, Petrášová et al. (2011) found the same STs in both types of host, but sequence analysis suggested that NHPs did not constitute a reservoir for human infection. Conversely, Yoshikawa et al. (2009) concluded that rhesus monkeys might serve as a reservoir for human ST2 infections in Nepal. Apart from this, very little is known about the distribution of STs in NHPs, and whether the potential differences reported among NHPs are linked to host species/ genera and/or behaviour/geography.

Analysis of data obtained by multilocus sequence typing (MLST) of the Blastocystis mitochondriallike organelle genome has shown that many ST3 strains from NHPs are distinct from those found in 
humans, indicating that cryptic host specificity exists (Stensvold et al. 2012), at least in this ST. We also demonstrated that specific polymorphisms in SSU rDNA sequences constitute valid surrogate markers for the intra-ST diversity detected by MLST (Stensvold et al. 2012). In the present study we have analysed SSU-rDNAs from NHP Blastocystis samples to (i) identify the range of STs hosted by NHPs, (ii) investigate the existence of any potentially host-specific STs or alleles, and (iii) explore whether NHP characteristics (taxon, geography) are linked to STs or alleles. We also compared NHP Blastocystis sequences to human Blastocystis sequences in order to investigate whether host-specific alleles can be identified in STs other than ST3.

\section{MATERIALS AND METHODS}

\section{Samples}

Fecal samples for analysis were obtained from NHPs in zoos and sanctuaries in the UK, France, the Netherlands, Germany and Italy (Supplementary Table - in Online version only). Samples from wild primates were collected in Morocco and St Kitts. For some samples, genomic DNA was extracted directly from fresh or frozen stool using the QIAGEN DNA Stool Mini Kit (QIAGEN, Hilden, Germany) according to the recommendations of the manufacturer. Most samples, however, were subject to short-term in vitro culture using Robinson's medium (Clark and Diamond, 2002) prior to DNA extraction, which was carried out as follows. Cells were harvested by centrifugation, washed $\times 3$ in phosphate-buffered saline, and lysed in $0 \cdot 25 \% \mathrm{SDS} / 0 \cdot 1 \mathrm{M}$ EDTA ( $\mathrm{pH}$ 8). DNA was purified from lysates using the Puregene Core Kit A (QIAGEN, Hilden, Germany) according to the recommendations of the manufacturer.

\section{PCR and sequencing}

DNAs were submitted to barcoding (Scicluna et al. 2006). Briefly, the primers RD5 and BhRDr were used to amplify a PCR product of $c .600 \mathrm{bp}$. Products were purified using either the GeneJET ${ }^{\mathrm{TM}}$ PCR Purification Kit (Fermentas, York, UK) or SureClean (Bioline, London, UK) and analysed on an ABI 3730 capillary sequencer after sequencing with the BhRDr primer and BigDye v. 3.1 sequencing reagents (Applied Biosystems, Warrington, UK).

\section{Subtype and allele identification}

Blastocystis SSU-rDNAs from NHPs available in GenBank as of 1 January 2012 were downloaded and those spanning the barcode region were included in this study (Supplementary Table-in Online version only). Information on host species, species range, location of individual sampled, and GenBank accession no. was recorded where available (Supplementary Table). Current taxonomy and ecological information was obtained from the Primate Info Network (http://pin.primate.wisc.edu/). STs and SSU-rDNA alleles were assigned to sequences using BLAST searches of the NCBI GenBank database and the Blastocystis Sequence Typing Database (www.pubmlst.org/blastocystis) (Jolley and Maiden, 2010; Stensvold et al. 2012), using the 'Sequence Query' algorithm at the latter. The '18S allele' database is not currently part of MLST schemes, but enables discrimination of SSU-rDNA alleles. This offers more resolution than the subtyping system but less resolution than the MLST schemes (Stensvold et al. 2012). Hence, sequences corresponding to $400 \mathrm{bp}$ near the 5 '-end of the SSU rDNA are assigned to an ST and an allele in the database; each ST comprises at least 2 alleles. Importantly, ' $18 \mathrm{~S}$ allele' numbers are unique and assigned consecutively as new alleles are discovered irrespective of ST. The vast majority of the sequences met with submission requirements (sequence length and quality) and were submitted to the Blastocystis 18S database at www.pubmlst.org/ blastocystis.

In addition to sequences, some ST data for NHPs obtained by other techniques, primarily Sequence Tagged Site (STS) PCR amplification, are also available. These were included to give as complete a picture of the distribution as possible (Supplementary Table).

RESULTS AND DISCUSSION

\section{Subtype distribution}

Novel data were obtained for Blastocystis in 260 NHPs and were combined with 57 NHP Blastocystis sequences previously deposited in GenBank. The dataset includes sequences from 6 lemurs (Prosimii), 54 New World (NW) Monkeys (Ceboidea), 169 Old World (OW) Monkeys (Cercopithecoidea), 75 apes (Hominoidea) and 13 unidentified NHPs (Supplementary Table-in Online version only). Among the newly generated sample data, 20 sequence traces clearly represented mixed STs. Excluding these mixed ST infections, 79 sequences in the combined dataset belonged to ST1 (27\%), 65 to ST2 $(22 \%), 101$ to ST3 $(34 \%), 3$ to ST4 $(1 \%), 13$ to ST5 $(4 \%), 32$ to ST8 $(11 \%), 1$ to ST13 $(<1 \%)$ and 3 to ST15 (1\%).

The 169 OW monkeys (Supplementary Table) include representatives of 12 genera: Allenopithecus $(n=4), \quad$ Cercocebus $(n=3), \quad$ Cercopithecus $(n=8)$, Chlorocebus $(n=42)$, Colobus $(n=21)$, Erythrocebus $(n=4), \quad$ Lophocebus $\quad(n=4), \quad$ Macaca $\quad(n=38)$, Mandrillus $(n=29)$, Papio $(n=4)$, Semnopithecus $(n=6)$ and Trachypithecus $(n=6)$. The natural species 
range of these NHPs is limited to Africa, apart from Trachypithecus, Semnopithecus and most Macaca species, which are native to Asia. In addition to West Africa, Chlorocebus sabaeus is also found on some West Indian Islands. Clear mixed infections were seen in 15/169 (9\%) cases. Excluding mixed ST infections, the sequences from the remaining $154 \mathrm{OW}$ monkey Blastocystis belonged to ST1 (33\%), ST2 (21\%), ST3 (43\%), ST5 (1\%), ST8 (1\%) and ST13 (1\%). Only ST1, ST2 and ST3 were seen in mixed infections. Interestingly, all three infections belonging to ST5 and ST8 were from species of Trachypithecus, and hence these two STs were not detected in OW monkey species native to the African continent.

Apes include Pan troglodytes $(n=27)$, Pan paniscus $(n=4)$ Gorilla gorilla $(n=11)$, Pongo pygmaeus $(n=15), \quad$ Hylobates spp. $(n=6)$, Nomascus spp. $(n=6)$ and Symphalangus syndactylus $(n=6)$. A total of 4/75 apes were positive for more than one ST (Supplementary Table). Excluding these mixed ST infections, sequences from apes belonged to either ST1 (21\%), ST2 (30\%), ST3 (24\%), ST5 (15\%), ST8 (7\%) or ST15 (3\%). As for OW monkeys, ST8 was seen only in apes native to SE Asia, namely in S. syndactylus $(n=3)$, Hylobates lar $(n=1)$ and Hylobates lar/agilis $(n=1)$, all of which are almost exclusively arboreal. Among the more numerous hosts sampled, chimpanzees and orangutans were positive for ST1, ST2, ST3 and ST5, while gorillas were positive for the same STs apart from ST1 (Supplementary Table).

New World monkeys (Ceboidea) comprise Alouatta caraya $(n=5)$, Ateles spp. $(n=6)$, Callithrix jacchus $(n=1)$, Lagothrix lagotricha $(n=39)$, Leontopithecus rosalia $(n=1)$, Pithecia pithecia $(n=1)$ and Saguinus labiatus $(n=1)$. One case of mixed ST infection was detected. Forty-five per cent of sequences from the NW monkeys belonged to ST8, while $19 \%$ belonged to ST1, $13 \%$ to ST2 and $21 \%$ to ST3; 1 ST4 sequence $(2 \%)$ was seen. Nineteen of 22 ST8 sequences were from L. lagotricha, and the remaining 3 were from $A$. caraya, the only $\mathrm{ST}$ found in the latter host. It is notable that the same allele of ST8 was found in A. caraya from zoos in 2 different countries.

Only 5 species of Prosimii and 6 samples were available for inclusion, and 4 different STs were seen: a Hapalemur aureus had ST1, 1 Lemur catta had ST2 and another ST4, a Varecia rubra had ST4, a Varecia variegata had ST8 and a Eulemur fulvus rufus had ST15 (Supplementary Table). Few other samples of Blastocystis from lemurs have been investigated, but when non-barcode data are included the range of STs is extended to include ST10 also. This range is surprisingly broad yet ST3 is notable by its absence, although the small number of samples precludes any conclusions at this stage. Only studies of samples from wild lemurs in Madagascar can confirm whether all the STs detected are naturally occurring in this host group.

Humans have been shown to primarily host ST1-ST4; infections with several other STs are suspected of being the result of zoonotic transmission (Stensvold et al. 2008, 2009). It is perhaps not surprising, therefore, that more than $80 \%$ of the NHP Blastocystis sequences analysed here belong to ST1-ST3, and that these STs are seen in NHPs from all geographical regions.

\section{NHP subtypes and alleles}

Assignment of alleles was possible for 212 sequences; in the remaining cases allele assignment was impossible either due to the data lacking the barcode region (e.g. where the sequence data available were from other regions in the SSU-rRNA gene), partial sequence data (sequences not covering the whole region necessary for allele assignment), mixed ST infection, or one or more ambiguous/mixed base calls. A total of 36 different alleles were identified across all STs; 6/36 alleles were represented by 1 sequence only and in some cases these represented sequences obtained from cloned DNA. Distinctions may be necessary when comparing allele data obtained by sequencing cloned PCR products vs direct sequencing of the PCR product itself, since the latter sequence will represent the dominant allele in the original sample. Not surprisingly, most variation was seen in ST1-ST3: ST1 exhibited 6 alleles, ST2 exhibited 7, and ST3 a total of 18 alleles, a distribution proportional to the number of sequences assigned to each ST. In comparison, only 2 alleles each were seen in ST5 and ST8.

Generally, host specificity and ST data generated from material collected from zoo animals should be interpreted with caution since species may be infected with STs to which they are exposed in captivity but which may not be circulating in natural populations. Many of the primates examined in this study are from zoos and so we cannot be sure that the STs and alleles of Blastocystis they carry would be found in wild representatives of the same species. Studies using DNA from wild NHPs will be needed to confirm that the STs detected in captive animals are representative. However, the data nevertheless still indicate the subtypes to which the host species are susceptible and are therefore of use.

Some findings do suggest that the captive infections reflect the situation in the wild. Of the 42 sequences from Chlorocebus spp., 10 belonged to Chlorocebus pygerythrus and Chlorocebus aethiops from Tanzania and zoos in the Netherlands, Germany and Japan, while the rest were from Chlorocebus sabaeus, which was introduced to St Kitts in the 17 th century. Both the wild and captive species host ST1, ST2 and ST3, while allele 14 (ST2) was the most common sequence found in the St Kitts 
Table 1. Overview of Blastocystis sp. STs detected in major groups of primates

\begin{tabular}{|c|c|c|c|c|c|c|c|c|c|c|c|c|c|c|c|}
\hline \multirow[b]{2}{*}{ Host family/genus } & \multicolumn{13}{|c|}{ Blastocystis sp. } & \multirow[b]{2}{*}{ Total } & \multirow[b]{2}{*}{ References $^{\mathrm{a}}$} \\
\hline & ST1 & ST2 & ST3 & ST4 & ST5 & ST6 & ST7 & ST8 & ST9 & ST10 & ST13 & $\mathrm{ST} 15^{\mathrm{b}}$ & $\begin{array}{l}\text { Mixed/ } \\
\text { unknown } \\
\text { ST }\end{array}$ & & \\
\hline $\begin{array}{l}\text { Chimpanzee } \\
\text { (including } \\
\text { Bonobo }(n=4) \text { ) }\end{array}$ & 24 & 11 & 20 & - & 12 & - & - & - & - & - & - & 1 & 3 & 71 & $1,2,4,5,12$ \\
\hline Gorilla & - & 10 & 2 & - & 4 & - & - & - & - & - & - & - & 1 & 17 & 1,12 \\
\hline Orangutan & 10 & 6 & 5 & - & 2 & - & - & - & - & - & - & - & - & 23 & $1-4,6-7,12,13$ \\
\hline Gibbons & 12 & 8 & 3 & - & 2 & - & - & 7 & - & - & - & 1 & - & 33 & $1-4,6-7,12,13$ \\
\hline $\begin{array}{l}\text { Non-human } \\
\text { Hominoids }\end{array}$ & 46 & 35 & 30 & - & 20 & - & - & 7 & - & - & - & 2 & 4 & 144 & \\
\hline Baboons & 3 & - & 2 & - & - & - & - & - & - & - & - & - & 2 & 7 & $1,6,7$ \\
\hline Mandrill/drill & 19 & - & 9 & - & - & - & - & - & - & - & - & - & 5 & 33 & $1-4,6,7,13$ \\
\hline Macaques & 17 & 19 & 27 & - & - & - & - & - & - & - & - & - & 1 & 64 & $1-3,6,7-10,14$ \\
\hline Vervet monkey & 12 & 24 & 11 & - & 2 & - & - & - & - & - & - & - & - & 49 & $1,2,3,5,6$ \\
\hline Colobus monkey & 14 & 2 & 6 & - & - & - & - & - & - & - & 5 & - & 3 & 30 & $1,5,11$ \\
\hline Mangabeys & 1 & 1 & 4 & - & - & - & - & - & - & - & - & - & 1 & 7 & 1,13 \\
\hline $\begin{array}{l}\text { Allen's swamp } \\
\text { monkey }\end{array}$ & - & - & 4 & - & - & - & - & - & - & - & - & - & - & 4 & 1 \\
\hline Guenons & 3 & 1 & 4 & - & - & - & - & - & - & - & - & - & 6 & 14 & $1-4,13$ \\
\hline Patas monkey & 1 & - & 3 & - & - & - & - & - & - & - & - & - & - & 4 & 1 \\
\hline Langurs/lutungs & 6 & 1 & 3 & - & 2 & - & - & 1 & - & - & - & - & 1 & 14 & $1-4$ \\
\hline Cercopithecoids & 76 & 48 & 73 & - & 4 & - & - & 1 & - & - & 5 & - & 19 & 226 & \\
\hline Woolly monkey & 5 & 5 & 9 & 1 & - & - & - & 19 & - & - & - & - & - & 39 & $1,12,14$ \\
\hline Howler monkey & - & - & - & - & - & - & - & 5 & - & - & - & - & - & 5 & 1 \\
\hline Spider monkey & 3 & 2 & - & - & - & - & _- & - & - & - & - & - & 1 & 6 & 1 \\
\hline Marmoset & - & - & 2 & - & - & - & - & - & - & - & - & - & - & 2 & 1,12 \\
\hline Tamarin $^{c}$ & 1 & - & 1 & - & - & - & - & - & - & - & - & - & - & 2 & 1 \\
\hline Saki monkey & 1 & - & - & - & - & - & - & - & - & - & - & - & - & 1 & 1 \\
\hline Ceboids & 10 & 7 & 12 & 1 & - & - & - & 24 & - & - & - & - & 1 & 55 & \\
\hline Lemurs & 4 & 2 & - & 4 & - & - & - & 3 & - & 2 & - & 1 & - & 16 & $1,2,3,6,7,12,13$ \\
\hline $\begin{array}{l}\text { NHP primates } \\
\text { total }\end{array}$ & $\begin{array}{l}136 \\
(30 \cdot 8 \%)\end{array}$ & $\begin{array}{l}92 \\
(20 \cdot 9 \%)\end{array}$ & $\begin{array}{l}115 \\
(26 \cdot 1 \%)\end{array}$ & $\begin{array}{l}5 \\
(1 \cdot 1 \%)\end{array}$ & $\begin{array}{l}24 \\
(5 \cdot 4 \%)\end{array}$ & $\begin{array}{l}- \\
-\end{array}$ & - & $\begin{array}{l}35 \\
(7 \cdot 9 \%)\end{array}$ & - & $\begin{array}{l}2 \\
(0 \cdot 4 \%)\end{array}$ & $\begin{array}{l}5 \\
(1 \cdot 1 \%)\end{array}$ & $\begin{array}{l}3 \\
(0 \cdot 7 \%)\end{array}$ & $\begin{array}{l}24 \\
(5 \cdot 4 \%)\end{array}$ & $\begin{array}{l}441 \\
(100 \%)\end{array}$ & \\
\hline Humans total & $\begin{array}{l}882 \\
(27 \cdot 8 \%)\end{array}$ & $\begin{array}{l}343 \\
(10 \cdot 8 \%)\end{array}$ & $\begin{array}{l}1399 \\
(44 \cdot 1 \%)\end{array}$ & $\begin{array}{l}318 \\
(10 \cdot 0 \%)\end{array}$ & $\begin{array}{l}9 \\
(0 \cdot 3 \%)\end{array}$ & $\begin{array}{l}89 \\
(2 \cdot 8 \%)\end{array}$ & $\begin{array}{l}118 \\
(3 \cdot 7 \%)\end{array}$ & $\begin{array}{l}10 \\
(0 \cdot 3 \%)\end{array}$ & $\begin{array}{l}3 \\
(0 \cdot 1 \%)\end{array}$ & $\begin{array}{l}- \\
-\end{array}$ & $\begin{array}{l}- \\
-\end{array}$ & $\begin{array}{l}- \\
-\end{array}$ & $\begin{array}{l}225 \\
\text { NA }\end{array}$ & $\begin{array}{l}3171^{\mathrm{d}} \\
(100 \%)\end{array}$ & 15 \\
\hline
\end{tabular}

a 'Present study' refers to the Supplementary Table and includes data from other studies as well as the sequences generated here. However, in some published studies nucleotide sequences for some samples have not been made publically available, with only representative sequences being deposited in GenBank. For instance, in the study by Petrášová et al. (2011), only 12 sequences were submitted to GenBank and were included in the Supplementary Table while the remaining data are included in this table as reference no. 5 (see below). In some other studies STS primers (Yoshikawa et al. 1998) have been used for ST identification rather than sequencing and so are not in the Supplementary Table but are included here. References: $1=$ Present study, $2=$ Abe $e t$ al . (2003), 3= Abe (2004), 4=Yoshikawa et al. (2004), 5= Petrášová et al. (2011), 6= Parkar et al. (2007), $7=$ Parkar et al. (2010), 8=Yoshikawa et al. (2009), 9=Yoshikawa et al. (1998), $10=$ Yoshikawa et al. (2003), $11=$ Teichroeb et al. (2009), $12=$ Stensvold et al. (2009), 13= Santín et al. (2011), 14= Scicluna et al. (2006), 15=Alfellani et al. (2013).

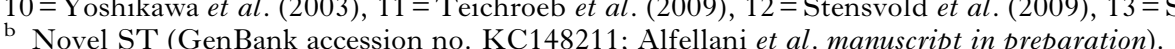

c Including Leontopithecus.

c Including Leontopithecus.
d This total does not include mixed/unknown infections. 
monkeys and was also found in a wild Tanzanian monkey by Petrášová et al. (2011). Similarly, in Macaca sylvanus from the wild in Morocco and from German and UK zoos only ST1-ST3 were identified, and some of the alleles were present in both wild and captive animals (Supplementary Table).

ST4 appears to be rare in NHPs (Table 1) since it has been reported only in 1 woolly monkey (present study) and in captive lemurs (Stensvold et al. 2009; Santín et al. 2011). The woolly monkey ST4 sequence exhibited allele 133. Interestingly, this allele has not previously been reported, yet it was shared by the woolly monkey in the UK and in a ruffed lemur from a German zoo. The fact that it is a new allele reduces the possibility of sample mix-up, laboratory contamination or exposure in captivity to this ST. Hence the finding indicates that these 2 species may be natural hosts of ST4. It remains to be seen whether ST4 can be found in other NHP hosts and in wild representatives of these species. Previously, only 2 ST4 alleles have been found in humans (42 and 94), one of which (94) appears to be extremely rare; both also occur in rodents (Stensvold et al. 2012).

ST5 is seen mainly in African apes, but also in a couple of Asian cercopithecoids (Table 1). It has not been found in NW monkeys, but since ST5 has not been reported in any South American hosts, it is too early to speculate as to whether the absence of ST5 in South American monkeys is due to host specificity or to geographical restriction.

ST13 was first reported by Parkar et al. (2010) in a quokka (a marsupial) but their sequence lacked the barcode region. A sequence from a wild Colobus guereza included in the study by Petrášová et al. (2011) was assigned by those authors to ST5, but comparison with a complete ST13 sequence (Alfellani et al. manuscript in preparation) showed them to be $98-99 \%$ identical. This has enabled reassignment of this Colobus sequence to ST13 and means that this ST is not restricted to Australia or marsupials.

ST15 is a newly discovered ST that is quite divergent from the others found in primates (Alfellani et al. manuscript in preparation). Nevertheless it has been found in both apes and a prosimian, and both African and Asian hosts, suggesting that it is quite widespread both geographically and in host range.

\section{Comparison of human and NHP Blastocystis}

In the total analysis (Table 1), ST1 was the most common ST identified, being found in 136 NHPs (excluding mixed infections); this is the second most common ST in humans (Table 1). While allele 4 accounts for over $95 \%$ of all human ST1s, non-human primate ST1s belongs to at least 6 alleles (Supplementary Table), with a slight preponderance of alleles 1 and 2 and with, as yet, no apparent link to

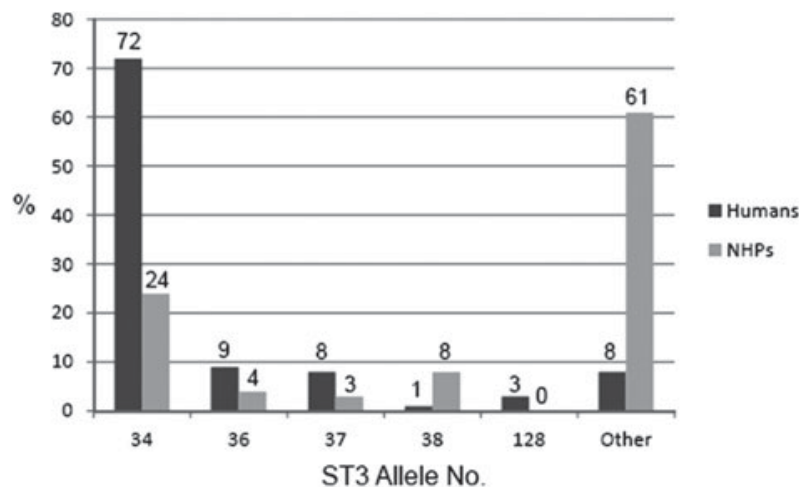

Fig. 1. ST3 SSU allele distribution in humans and NHPs. NHP data are from this study, and human data were retrieved from the isolate database available at www.pubmlst.org/blastocystis as of 1 June 2012. While alleles 34, 36, 37, 38 and 128 account for 170/185 (92\%) of human ST3 infections, NHP ST3 is assigned to 1 of these 5 alleles in only $39 \%$ of the samples, and many alleles found in NHPs have not so far been identified in humans.

NHP host species. During the collection of data for this study, we came across 2 ST1 isolates from zoo keepers (data not shown); one was assigned to allele 2 and another to allele 6 , both of which are extremely rare in humans and likely are examples of zoonotic transmission from zoo animals, most likely NHPs. Conversely, human and NHP ST2s appear to be comparable, both being distributed across the same 6-7 alleles.

The situation in ST3 appears somewhat analogous to ST1. This ST is the most common ST found in studies of human Blastocystis (Table 1). We recently demonstrated that while humans and NHPs can host some of the same alleles of ST3, other ST3 alleles appear to be NHP specific (Stensvold et al. 2012). The present study further supports this finding. The most common ST3 allele in humans is allele $34(72 \%)$, but this allele accounts for only $24 \%$ of NHP ST3. While $92 \%$ of human ST3 belongs to one of alleles $34,36,37,38$ or 128 , only $39 \%$ of NHP ST3 belongs to these 5 alleles (Fig. 1). No single ST3 allele appears to dominate among NHPs or any subset thereof.

Of the rarer STs, ST6 and ST7 are occasionally found in humans but are generally considered 'avian subtypes' and so of zoonotic origin in humans. Interestingly, so far there is no evidence of these STs in NHPs despite the fact that NHPs will certainly be exposed to bird feces at least as much as humans. In contrast, human ST8 infections are rarely reported except in zookeepers (Scicluna et al. 2006; unpublished observations) but are common in some NHPs (Table 1) suggesting that zoonotic transmission is likely occurring. ST9 has not been reported from NHPs, but as it is very rare in humans any conclusions on host specificity are premature. 
Three STs, ST10, ST13 and ST15, have been found in NHPs but not, to date, in humans. Given that subtyping of human Blastocystis in regions where NHPs with these infections are found is limited or absent it would be inappropriate to speculate on the host range of these STs at this stage. However, it is worth noting that ST10 is common in cattle and sheep in several countries where human sampling has been undertaken without any human infections with this ST being detected.

\section{CONCLUSION}

This study is the first paper to generate and analyse a large sample of Blastocystis STs in NHPs. At first glance it appears that there is a considerable overlap between the range of STs and their relative distribution in humans and NHPs. A study of the more subtle but consistent differences at SSU allele level, however, has enabled us to identify cryptic host specificity of alleles in at least the 2 most common STs, ST1 and ST3. In addition, some initial suggestions of host restriction of STs and alleles have been obtained but further sampling is needed. Transmission from NHP to human may occur mainly in zoos and animal sanctuaries as demonstrated in this and a recent study (Stensvold et al. 2012). However, no studies of alleles in humans and wild NHPs in the same region have been undertaken so the role of NHPs in zoonotic transmission is unresolved. The study of STs and alleles in other host animals is key to completing our understanding of Blastocystis epidemiology.

\section{ACKNOWLEDGEMENTS}

Bonaventura (Bino) Majolo and Richard McFarland are thanked for providing the Morocco macaque samples, Simone Caccio for providing several DNA samples, Ghislaine Sayers of Paignton Zoo for many primate stool samples and the staff of the Diagnostic Parasitology Laboratory, LSHTM for culture lysates. Samples from C. sabaeus were from the Behavioural Science Foundation on St Kitts and were obtained with technical assistance from Binh Ho-Thanh and Brandon Weaver.

\section{FINANCIAL SUPPORT}

Much of this work formed part of the PhD thesis of MAA, who was supported by the Libyan Government, and some formed part of an MSc project submitted by DT-M.

\section{REFERENCES}

Abe, N. (2004). Molecular and phylogenetic analysis of Blastocystis isolates from various hosts. Veterinary Parasitology 120, 235-242.
Abe, N., Wu, Z. and Yoshikawa, H. (2003). Molecular characterization of Blastocystis isolates from primates. Veterinary Parasitology 113, 321-325.

Alfellani, M. A., Stensvold, C. R., Vidal-Lapiedra, A., Onuoha, E. S. U., Fagbenro-Beyioku, A.F. and Clark, C. G. (2003). Variable geographic distribution of Blastocystis subtypes and its potential implications. Acta Tropica 126, 11-18.

Clark, C. G. and Diamond, L.S. (2002). Methods for cultivation of luminal parasitic protists of clinical importance. Clinical Microbiology Reviews 15, 329-341.

Fayer, R., Santin, M. and Macarisin, D. (2012). Detection of concurrent infection of dairy cattle with Blastocystis, Cryptosporidium, Giardia, and Enterocytozoon by molecular and microscopic methods. Parasitology Research 111, 1349-1355.

Jolley, K. A. and Maiden, M. C. (2010). BIGSdb: scalable analysis of bacterial genome variation at the population level. BMC Bioinformatics 11, 595.

Parkar, U., Traub, R. J., Kumar, S., Mungthin, M., Vitali, S., Leelayoova, S., Morris, K. and Thompson, R. C. (2007). Direct characterization of Blastocystis from faeces by PCR and evidence of zoonotic potential. Parasitology 134, 359-367.

Parkar, U., Traub, R. J., Vitali, S., Elliot, A., Levecke, B., Robertson, I., Geurden, T., Steele, J., Drake, B. and Thompson, R. C. (2010). Molecular characterization of Blastocystis isolates from zoo animals and their animal-keepers. Veterinary Parasitology 169, 8-17.

Petrášová, J., Uzlíková, M., Kostka, M., Petrželková, K. J., Huffman, M. A. and Modrý, D. (2011). Diversity and host specificity of Blastocystis in syntopic primates on Rubondo Island, Tanzania. International Fournal for Parasitology 41, 1113-1120.

Santín, M., Gómez-Muñoz, M. T., Solano-Aguilar, G. and Fayer, R. (2011). Development of a new PCR protocol to detect and subtype Blastocystis spp. from humans and animals. Parasitology Research 109, 205-212.

Scicluna, S. M., Tawari, B. and Clark, C. G. (2006). DNA barcoding of Blastocystis. Protist 157, 77-85.

Stensvold, C. R., Suresh, G. K., Tan, K. S. W., Thompson, R. C.A., Traub, R. J., Viscogliosi, E., Yoshikawa, H. and Clark, C. G. (2007). Terminology for Blastocystis subtypes - a consensus. Trends in Parasitology 23, 93-96.

Stensvold, C. R., Arendrup, M. C., Nielsen, H. V., Bada, A. and Thorsen, S. (2008). Symptomatic infection with Blastocystis sp. subtype 8 successfully treated with trimethoprim-sulfamethoxazole. Annals of Tropical Medicine and Parasitology 102, 271-274.

Stensvold, C. R., Alfellani, M. A., Nørskov-Lauritsen, S., Prip, K., Victory, E. L., Maddox, C., Nielsen, H. V. and Clark, C. G. (2009) Subtype distribution of Blastocystis isolates from synanthropic and zoo animals and identification of a new subtype. International fournal for Parasitology 39, 473-479.

Stensvold, C. R., Alfellani, M. and Clark, C. G. (2012). Levels of genetic diversity vary dramatically between Blastocystis subtypes. Infection, Genetics and Evolution 12, 263-273.

Teichroeb, J. A., Kutz, S. J., Parkar, U., Thompson, R. C and Sicotte, P. (2009). Ecology of the gastrointestinal parasites of Colobus vellerosus at Boabeng-Fiema, Ghana: possible anthropozoonotic transmission. American Fournal of Physical Anthropology 140, 498-507.

Yoshikawa, H., Nagano, I., Wu, Z., Yap, E. H., Singh, M. and Takahashi, Y. (1998). Genomic polymorphism among Blastocystis hominis strains and development of subtype-specific diagnostic primers. Molecular and Cellular Probes 12, 153-159.

Yoshikawa, H., Abe, N. and Wu, Z. (2003). Genomic polymorphism among Blastocystis isolates and development of PCR-based identification of zoonotic isolates. Fournal of Eukaryotic Microbiology 50, 710-711.

Yoshikawa, H., Abe, N. and Wu, Z. (2004). PCR-based identification of zoonotic isolates of Blastocystis from mammals and birds. Microbiology 150, 1147-1151.

Yoshikawa, H., Wu, Z., Pandey, K., Pandey, B. D., Sherchand, J. B., Yanagi, T. and Kanbara, H. (2009). Molecular characterization of Blastocystis isolates from children and rhesus monkeys in Kathmandu, Nepal. Veterinary Parasitology 160, 295-300. 